S. R. Bowman and L. O'Carroll

Nagoya Math. J.

Vol. 102 (1986), 155-161

\title{
TENSOR PRODUCTS AND LOCALIZATIONS OF ALGEBRAS
}

\author{
S. R. BOWMAN AND L. O'CARROLL
}

In a recent paper [5], it was shown that the tensor product of a finite number of fields over a common subfield satisfies the property that each localization at a prime ideal is a primary ring (in the sense that a zerodivisor is in fact a nilpotent element).

In the first section of this paper, we exploit the properties of associated primes and of flat extensions so as to generalize the above result to zerodimensional algebras; a simple example shows that this is the best one can hope for. The converse situation is also investigated.

In the second section, a result of Grothendieck [1] is used to answer an open question in [2], so completing the programme of generalizing the work of Trung [7] and Nagata [4] on a conjecture of O'Carroll and Qureshi [6].

During work on this research, the first author was supported by an S.E.R.C. postgraduate studentship at the University of Edinburgh.

\section{§1. Locally primary rings}

In this paper, all rings are commutative with identity element. In fact only algebras over a field, $k$ say, are considered, and all tensor products are taken over $k$. For the properties of flat extensions and associated primes we refer to [3, Chapters 2 and 3]. The basic theory of the tensor product of algebras is given in [9, Chapter III]; a brief discussion of the interaction with the theory of flat extensions is given in [5].

A primary ring is one in which each zero-divisor is a nilpotent element. A ring $R$ is called locally primary if, for each prime ideal $P$ in $R, R_{P}$ is a primary ring. In [5, Theorem 3] it is shown that the tensor product of a finite number of fields over a common subfield is a locally primary ring. Our first result examines the converse situation.

Proposition 1. Let $R_{1}, \cdots, R_{n}$ be algebras over a field $k$, and suppose

Received March 1, 1985. 
that $R_{1} \otimes \cdots \otimes R_{n}$ is a locally primary ring. Then each $R_{i}$ is also a locally primary ring.

Proof. We may restrict our attention to $R_{1}$, which we refer to as $R$; let $S=R_{1} \otimes \cdots \otimes R_{n}$. Consider a prime ideal $P$ in $R$. Since, by base change, $S$ is a faithfully flat extension of $R$, there exists a prime ideal $Q$ in $S$ which contracts to $P$ in $R$. Regarding $S$ as an $R$-module we have $R_{P} \subseteq S_{P}$, and we also have the natural map $S_{P} \rightarrow S_{Q}$. Let $r \in R$. Since $S$ is a flat extension of $R, 0:_{S} r=\left(0:_{R} r\right) S$. Hence if $0:_{S} r \not \subset Q$, then $0:_{R} r \not P$. It follows that $R_{P}$ is isomorphically embedded in $S_{Q}$ by the natural maps given above. By hypothesis, $S_{Q}$ is a primary ring. It follows that $R_{P}$ is also a primary ring.

However, the following example shows that the converse of Proposition 1 does not hold in general.

ExAmple. Let $R_{1}=\boldsymbol{Q}[X, Y] /\left(X^{2}-2 Y^{2}\right)$, and let $R_{2}=\boldsymbol{Q}(\sqrt{2})$. Since $R_{1}$ and $R_{2}$ are domains, they are primary, and so locally primary, rings in a trivial way. However the ring

$$
S:=R_{1} \otimes_{Q} R_{2}=Q(\sqrt{2})[X, Y] /\left(X^{2}-2 Y^{2}\right)
$$

is not locally primary, since the maximal ideal $(X, Y) S$ contains two distinct minimal prime ideals $(X-\sqrt{2} Y) S$ and $(X+\sqrt{2} Y) S$. (Note that a primary ring contains a unique minimal prime ideal, consisting of all the zero-divisors.)

Note also that $\operatorname{dim} R_{2}=0$, but that $\operatorname{dim} R_{1}=1$.

We now wish to generalize [5, Theorem 3] in such a way as to provide a partial converse to Proposition 1; a zero-dimensional ring is of course locally primary. First we have two simple preliminary lemmas.

Lemma 2. Let $T$ be a zero-dimensional algebra, over a field $k$, which is also a local ring, and let $F$ be a finite subset of $T$. Then $F$ is contained in a zero-dimensional subalgebra $S$ of $T$, where $S$ consists of a localization of an affine subalgebra of $T$ at a prime ideal.

Proof. Let $A=k[F]$ and let $S=A_{P}$, where $P$ is the restriction to $A$ of the maximal ideal of $T$. Since $P$ consists of nilpotent elements, it is the unique minimal prime ideal of $A$. Moreover, $A \backslash P$ lies in the group of units of $T$, and in particular $A \backslash P$ consists of non-zero-divisors in $A$. Hence $F \subseteq A \subseteq S \subseteq T$, and the result follows. 
Lemma 3. Let $A$ be a Noetherian ring. Then $A$ is locally primary if and only if, for each prime ideal $P$ in $A$,

$$
\{Q \in \operatorname{Spec} A \mid Q \subseteq P\} \cap \operatorname{Ass}_{A}(A)
$$

is a singleton.

Proof. Let $P$ be a prime ideal in $A$. Now $A_{P}$ is a primary ring if and only if $\operatorname{Ass}_{A_{P}}\left(A_{P}\right)$ is a singleton. But by [3, Lemma, p. 50], $\operatorname{Ass}_{A_{P}}\left(A_{P}\right)$ contracts in $A$ to $\{Q \in \operatorname{Spec} A \mid Q \subseteq P\} \cap \operatorname{Ass}_{A}(A)$, and the result follows.

We now come to the main result of this section.

Theorem 4. Let $R_{1}, \cdots, R_{n}$ be zero-dimensional algebras, over a field k. Then $R_{1} \otimes \cdots \otimes R_{n}$ is a locally primary ring.

Proof. Let $R=R_{1} \otimes \cdots \otimes R_{n}$, and let $P$ be a prime ideal in $R$. For $i=1, \cdots, n$, let $P_{i}$ denote the restriction of $P$ to $R_{i}$, and let $R_{i}^{\prime}$ denote the localization of $R_{i}$ at $P_{i}$. (Each $R_{i}^{\prime}$ is a zero-dimensional local ring.) Then $R_{P}$ is a localization, at a prime ideal, of the ring $R_{1}^{\prime} \otimes \cdots \otimes R_{n}^{\prime}$. Hence, without loss of generality, we assume in addition that each $R_{i}$ is a local ring.

To prove that $R_{P}$ is a primary ring, it suffices to show that a zerodivisor of the form $x / 1 \in R_{P}$ (where $x \in R$ ) is nilpotent. Thus, consider $x \in R$ such that $s x y=0$ for some $y \in R$ and $s \in R \backslash P$, with $A_{A n n} y \subseteq P$; we wish to show that $A_{n} x^{n} \not \subset P$, for some positive integer $n$. Now each of $s, x$ and $y$ consists of a finite sum of tensors of the form $r_{1} \otimes \cdots \otimes r_{n}$, where $r_{i} \in R_{i}(i=1, \cdots, n)$. For each such $i$, let $F_{i}$ denote the finite set of the $r_{i}$ which occur in this way, and let $S_{i}$ be the corresponding subalgebra of $R_{i}$ given by Lemma 2 (with $R_{i}$ playing the role of $T$ ).

Let $S=S_{1} \otimes \cdots \otimes S_{n}$. Then $S$ and each of the $S_{i}$ are Noetherian (in fact, $S$ is the localization of an affine algebra over $k$ ), and $s, x$ and $y$ lie in $S$; moreover, each $S_{i}$ is a local, zero-dimensional, ring. Let $Q=$ $P \cap S$. Then $A_{n} n_{s} y \subseteq Q$, and if $A_{A n n} x^{n} \not \subset Q$ for some positive integer $n$, then $\operatorname{Ann}_{R} x^{n} \not \subset P$. Hence, without loss of generality, we assume also that $R$ and each of the $R_{i}$ are Noetherian. Recall that each $R_{i}$ is also local and zero-dimensional, with maximal ideal $P_{i}$ (using the same notation as before).

For $i=1, \cdots, n$, let $F_{i}$ denote the field $R_{i} / P_{i}$, and let $D=F_{1} \otimes \cdots$ $\otimes F_{n}$. Now each $P_{i}$ consists of nilpotent elements, and hence so does $\left(P_{1}, \cdots, P_{n}\right) R$, Thus $\operatorname{Spec} R \approx \operatorname{Spec} D$, under the map induced by the 
natural projection $R \rightarrow D$.

Note that for $i=1, \cdots, n, F_{1} \otimes \cdots \otimes F_{i-1} \otimes R_{i} \otimes \cdots \otimes R_{n}$ is a flat $R_{i}$-algebra, by base change. By repeated use of [3, Corollary, p. 58] and [loc. cit., Proposition, p. 57], we deduce that $\operatorname{Ass}_{R}(R)$ corresponds to $\operatorname{Ass}_{D}(D)$ under the above isomorphism of spectra. However, by [5, Theorem 3], $D$ is locally primary. Thus $R$ is locally primary, by the criterion of Lemma 3.

Remarks. Similar ideas can be used to derive an alternative proof of [5, Theorem 3] from an extension of Vámos' result (cf. [8, Corollary 4]), viz. that in a finite tensor product of fields any prime ideal contains a unique minimal prime ideal. (This extension is a corollary of [5, Theorem 3], of course.) As above, one reduces to the case where the field extensions involved are finitely generated. It follows from [9, Corollary 1, p. 191] that the zero ideal in the tensor product of fields, which is now a Noetherian ring, is unmixed. The extension of the Vámos result, together with Lemma 3, now yield [5, Theorem 3].

Similarly, in any example like that given after Proposition 1 involving a Noetherian tensor product of two algebras which are domains, we know from [9, Corollary 1, p. 191] that the zero ideal in the tensor product will be unmixed. Hence (cf. Lemma 3), in constructing the example, the property to aim for is that some prime (and hence, some maximal) ideal should contain more than one minimal prime.

\section{§ 2. Localizations yielding equidimensional Hilbert rings}

Trung [7], and independently Nagata [4], confirmed a conjecture by O'Carroll and Qureshi [6] (which had been verified in special cases), that the tensor product of a finite number of fields over a common subfield, where at most one of the field extensions has infinite transcendence degree, is an equidimensional Hilbert ring. Subsequently this work was generalized by Howie and O'Carroll [2]. The result of this section answers a question left open in [2]; this completes the programme of generalizing the result of Trung and Nagata, without having to resort to the awkward device of shifting to a companion result in order to cover the case where the base field is finite. (See [2, §3, Remarks 3, 4 and 5].) We refer to [2] for a discussion of the connections between the various results mentioned above, and also Theorem 5 below.

(As a point of notation, if $D$ is a domain and $B$ is a subring of $D$, 
tr.d. $(D \mid B)$ denotes the transcendence degree of the quotient field of $D$ over that of $B$. Moreover, an equidimensional ring is one in which all maximal ideals have the same rank.)

Theorem 5. Let $D$ be a domain and let $B_{1}, \cdots, B_{n}$ be subrings of $D$, where $n \geq 1$. Suppose that $B_{1}$ is chosen so as to satisfy

$$
\text { tr.d. }\left(D \mid B_{1}\right)=\min _{i}\left\{\operatorname{tr.d.}\left(D \mid B_{i}\right)\right\} \text {. }
$$

Suppose further that

(i) $D$ is a finitely generated $B_{i}$-algebra, $1 \leq i \leq n$; and

(ii) $B_{2}, \cdots, B_{n}$ are integrally closed.

Let $S$ be the multiplicatively closed set generated by $\cup_{i}\left\{B_{i} \mid 0\right\}$.

Then $S^{-1} D$ is a Noetherian equidimensional Hilbert ring of dimension

$$
d=\operatorname{tr} . \text { d. }\left(D \mid B_{1}\right) \text {. }
$$

Proof. Let $B=\bigcap_{i} B_{i}$. In [2] (cf. Theorem 2 there) this result has been established in the case where $B$ is infinite. Thus we now suppose that $B$ is finite, and reduce to the former case. We may assume that $d \geq 1$.

Hence suppose that $B$ is a finite field, which has algebraic closure $K$, say. Consider the effect of applying $K \otimes-$, where the tensor product is taken over $B$.

Let $\mathfrak{q}$ be a minimal prime in $K \otimes D$. Now the extension $D \subseteq K \otimes D$ is (faithfully) flat, by base change, and $D$ is a domain. Thus $\mathfrak{q} \cap D=0$, by "Going Down".

Fix $i$ such that $1 \leq i \leq n$. Consider the extension $K \otimes B_{i} \subseteq K \otimes D$. Let

$$
\mathfrak{q}_{i}=\mathfrak{q} \cap\left(K \otimes B_{i}\right)
$$

and let

$$
\bar{D}=(K \otimes D) / \mathfrak{q} \quad \text { and } \quad \bar{B}_{i}=\left(K \otimes B_{i}\right) / \mathfrak{q}_{i},
$$

where we take $\bar{B}_{i} \subseteq \bar{D}, D \subseteq \bar{D}$ and $B_{i} \subseteq \bar{B}_{i}$ (since $\mathfrak{q}_{i} \cap B_{i}=0$ ).

Now the extension $B_{i} \subseteq K \otimes B_{i}$ is integral, with $B_{i}$ a domain and $\mathfrak{q}_{i} \cap B_{i}=0$; hence $\mathfrak{q}_{i}$ is a minimal prime in $K \otimes B_{i}$. Note also that $D \subseteq \bar{D}$ is an integral extension of domains, as is $B \subseteq \bar{B}_{i}$; thus tr.d. $\left(D \mid B_{i}\right)=$ tr.d. $\left(\bar{D} \mid \bar{B}_{i}\right)$. Moreover, $\bar{D}$ is a finitely generated $\bar{B}_{i}$-algebra. 
We are given an element $a \in D \backslash 0$, and we wish to produce a non-zero prime ideal $P^{\prime}$ in $D$ such that $a \notin P^{\prime}$ and $P^{\prime} \cap B_{i}=0,1 \leq i \leq n$; as in [2], this will suffice to prove the result. We consider $a$ as a non-zero element of $\bar{D}$.

The proof of [2, Theorem 2] shows (on replacing $F, B_{i}$ and $D$ there by $K, \bar{B}_{i}$ and $\bar{D}$ respectively) that

(i) there exists an element $x \in \bar{D}$ which is transcendental over each $\bar{B}_{i}$;

(ii) there exist an element $b \in K$ and a prime ideal $P$ in $\bar{D}$ such that $P$ is minimal over $(x-b) \bar{D}$;

(iii) $a \notin P$ and $P \cap \bar{B}_{1}[x]=(x-b) \bar{B}_{1}[x]$; and

(iv) for each $i=2, \cdots, n$, there exists an element $c_{i}$ in a maximal extension $C^{(i)}$ of $\bar{B}_{i}[x]$ (in $\bar{D}$ ) by a finite number of algebraically independent elements, with $C_{c_{i}}^{(i)} \subseteq \bar{D}_{c_{i}}$ an integral extension and $c_{i} \notin P$.

Fix $i$ such that $2 \leq i \leq n$, and let $P_{i}=P \cap \bar{B}_{i}$. Pull $P_{i}$ back to a prime ideal $\mathfrak{p}_{i}$ of $K \otimes B_{i}$ which contains $\mathfrak{q}_{i}$. By [1, Corollary (6.14.2)] (cf. [loc. cit., (5.13.5)]), $\left(K \otimes B_{i}\right)_{p_{i}}$ is an integrally closed domain. In particular, $\left(\mathfrak{q}_{i}\right)_{\mathfrak{p}_{i}}=0$, so $\left(K \otimes B_{i}\right)_{p_{i}}=\left(\bar{B}_{i}\right)_{P_{i}}$, and the latter is an integrally closed domain. Let $Q_{i}=P \cap C^{(i)}$. Then $\left(C^{(i)}\right)_{Q_{i}}$ is an integrally closed domain, being a localization of a polynomial extension of $\left(\bar{B}_{i}\right)_{P_{i}}$. Since $c_{i} \notin Q_{i}$, the extension $\left(C^{(i)}\right)_{Q_{i}} \subseteq \bar{D}_{Q_{i}}$ is integral (here we regard $\bar{D}$ as a $C^{(i)}$-module). By the "Cohen-Seidenberg" theorem, the extension $\left(C^{(i)}\right)_{Q_{i}} \subseteq \bar{D}_{Q_{i}}$ satisfies "Going Down". Moreover the extension $\bar{B}_{i}[x] \subseteq\left(C^{(i)}\right)_{Q_{i}}$ also satisfies "Going Down", since it is a flat extension, being a composite of flat extensions. Thus the composite extension $\bar{B}_{i}[x] \subseteq \bar{D}_{Q_{i}}$ satisfies "Going Down".

We are now in a position to apply the argument of [2, §2]. Note that $P$ survives in $\bar{D}_{Q_{i}}$. By the "Going Down" property, the prime $P \cap \bar{B}_{i}[x]$ is minimal over the ideal $(x-b) \bar{B}_{i}[x]$. But the latter is itself a prime ideal. We deduce that

$$
P \cap \bar{B}_{i}[x]=(x-b) \bar{B}_{i}[x], \quad 1 \leq i \leq n .
$$

Hence, for $i=1, \cdots, n, P_{i}=P \cap \bar{B}_{i}=0$; moreover $a \notin P$.

Finally, set $P^{\prime}=P \cap D$. Note that $a \notin P^{\prime}$, and that $P^{\prime} \neq 0$ by the "Incomparability" property in the integral extension $D \subseteq \bar{D}$ of domains. Moreover $P^{\prime} \cap B_{i}=0,1 \leq i \leq n$, and the result follows. 


\section{REFERENCES}

[1] A. Grothendieck, Éléments de Géométrie Algébrique, IV, I.H.E.S., 24 (1965).

[2] J. Howie and L. O'Carroll, Some localizations which are Hilbert rings, J. Algebra, 92 (1985), 366-374.

[ 3 ] H. Matsumura, Commutative algebra, Benjamin/Cummings, Reading, Massachusetts, 1980.

[4] M. Nagata, A conjecture of O'Carroll and Qureshi on tensor products of fields, Japan. J. Math., 10 (1984), 375-377.

[5] L. O'Carroll and M. A. Qureshi, Primary rings and tensor products of algebras, Math. Proc. Cambridge Philos. Soc., 92 (1982), 41-48.

[6] - On the tensor product of fields and algebraic correspondences, Quart. J. Math., (2) 34 (1983), 211-221.

[7] N. V. Trung, On the tensor product of extensions of a field, Quart. J. Math., (2) 35 (1984), 337-339.

[8] P. Vámos, On the minimal prime ideals of a tensor product of two fields, Math. Proc. Cambridge Philos. Soc., 84 (1978), 25-35.

[9] O. Zariski and P. Samuel, Commutative algebra, Vol. I, Springer-Verlag, New York, 1958.

Department of Mathematics, University of Edinburgh,

James Clerk Maxwell Building,

The King's Buildings,

Mayfield Road,

EDINBURGH EH9 ЗJZ. 